D.O.I.: $10.3895 / \mathrm{S} 1808-04482007000200012$

\title{
CONDIÇÕES DE SAÚDE E SEGURANÇA EM LABORATÓRIOS DE ENSAIOS DE MATERIAIS ELÉTRICOS: UMA FERRAMENTA DE ANÁLISE
}

\section{HEALTH AND SAFETY CONDITIONS IN ELECTRICAL MATERIALS LABORATORIES: AN ANALYSIS TOOL}

\author{
Fernando Gonçalves Amaral ${ }^{1}$; Laura Sanz Burmann²; Luis Antônio dos Santos Franz ${ }^{3}$; Patricia \\ Klaser Biasoli ${ }^{4}$; Fabiane Ely ${ }^{5}$ \\ ${ }^{1}$ Federal University of Rio Grande do Sul - UFRGS - Porto Alegre - Brasil \\ amaral@producao.ufrgs.br \\ ${ }^{2}$ Federal University of Rio Grande do Sul - UFRGS - Porto Alegre - Brasil laura@burmann.com.br \\ ${ }^{3}$ Federal University of Rio Grande do Sul - UFRGS - Porto Alegre - Brasil \\ franz@producao.ufrgs.br \\ ${ }^{4}$ Rio Grande Energia - RGE - Porto Alegre - Brasil pbiasoli@rge-rs.com.br \\ ${ }^{5}$ Rio Grande Energia - RGE - Porto Alegre - Brasil fely@rge-rs.com.br
}

\begin{abstract}
Resumo
As condições de trabalho em laboratórios de ensaios de materiais e equipamentos, utilizados e redes de distribuição de energia elétrica, expõem os operadores a riscos importantes concernentes à saúde e segurança. Nesse sentido, o objetivo deste estudo foi elaborar uma ferramenta participativa capaz de estudar estas condições, visando reduzir dos riscos de acidentes e doenças ocupacionais nestes laboratórios. A metodologia elaborada foi aplicada em quatro etapas: análise da demanda (consulta as supervisões e trabalhadores do laboratório e análise de indicadores de segurança e saúde); elaboração de uma ferramenta específica de análise com base nas normas existentes; aplicação desta no local objeto de estudo e com base nos resultados a indicação de possíveis soluções apresentadas na forma de um plano de ação de melhorias. Como resultado, a ferramenta mostrou-se eficaz para evidenciar situações passíveis de causar danos à saúde e à integridade física dos trabalhadores do laboratório analisado, sendo de aplicação e entendimento simples de parte dos laboratoristas.
\end{abstract}

Palavras-chave: Segurança, Saúde, Riscos, Laboratório, Eletricidade.

\section{Introdução}

A busca por melhorias nas condições inseguras é largamente difundida na literatura nos mais diversos processos, principalmente com foco na saúde e segurança no trabalho. A redução destas condições pode permitir então a diminuição de acidentes ou doenças, aumentando-se a qualidade e produtividade dos processos. As ações prevencionistas necessitam então fazer parte do conjunto de políticas de segurança nas empresas, pois elas tratam de algo indispensável ao pleno êxito das 
demais atividades, sendo também de inegável valor para a qualidade de vida dos trabalhadores da empresa (ZOCCHIO, 2000). Neste contexto, considerando atividades em que os trabalhadores estão expostos a vários riscos e, entre estes, aqueles caracterizados como graves e iminentes à integridade da vida, a implantação de uma política de saúde e segurança ocupacional deve ser mais criteriosa e abrangente ainda.

Em um laboratório de ensaios de materiais e equipamentos utilizados nas atividades de construção e manutenção de redes de distribuição de energia elétrica existem processos que expõem o trabalhador a riscos de acidentes. Estes podem ter como conseqüências lesões temporárias ou até mesmo a morte do trabalhador. Além disto, considerando-se exclusivamente os materiais e produtos manuseados, por si só são fonte de acidentes e/ou contaminação.

Esta situação é ainda agravada no momento em que os laboratórios prestadores de serviços, sofreram grandes transformações. O grande desenvolvimento tecnológico das inspeções e dos equipamentos utilizados nas análises, o grau de exigência dos clientes (internos e externos) e a competitividade do mercado, produziram modificações substanciais nos processos de trabalho. De acordo com Benoliel (1999), os laboratórios, em geral, vêm buscando a partir deste contexto, a implantação de um sistema de gestão da qualidade de seus processos.

$\mathrm{Na}$ literatura são encontrados normas e procedimentos relacionados à qualidade dos processos em laboratórios (INMETRO, 2003; OECD, 1998; ISO/IEC, 1997). Neste caso, a inexistência de tais procedimentos pode até mesmo prejudicar e invalidar os resultados provenientes das análises. No Brasil, a NBR 17025 apresenta os requisitos gerais para competências de laboratórios de ensaio e calibração. Esta aborda questões relacionadas ao controle, aprovação e emissão de documentos; análise crítica de pedidos, propostas e contratos; sub contratação de ensaios e calibração; aquisição de pedidos e suprimentos; atendimento ao cliente; reclamações; ações corretivas para problemas em ensaios; ações preventivas; auditorias internas; análises críticas pela direção; equipamentos; pessoal; acomodações e condições ambientais; entre outros tópicos.

No item específico sobre acomodações e condições ambientais, a NBR 17025 determina simplesmente que as instalações de laboratórios para ensaio e/ou calibração devem facilitar a realização dos ensaios. Para isso, o laboratório deve monitorar, controlar e registrar as condições ambientais, para que estes fatores não influenciem na qualidade dos resultados. A esterilidade biológica, poeira, distúrbios eletromagnéticos, radiação, umidade, alimentação elétrica, temperatura e níveis sonoros e de vibração devem receber atenção dos laboratoristas. Além disso, indica que os ensaios e/ou calibrações devem ser interrompidos quando as condições ambientais comprometerem os resultados (NBR 17025, 2005).

Assim, a qualidade dos processos de ensaios está intimamente ligada às condições de segurança e saúde em laboratórios. No entanto, a ênfase na legislação de saúde e segurança é menos 
específica que a abordagem de qualidade, apresentando uma lacuna na literatura; bem como, a existência de procedimentos de coleta e análise de dados específicos para tais fins. Porém, de acordo com Calça (1999), as Normas Regulamentadoras (NR) apresentam os pontos básicos e as condições de saúde e segurança admitidas para o trabalho humano. Logo, estudando-se legislações e manuais de qualidade para processos laboratoriais, em conjunto com as normas relacionadas diretamente com a segurança e saúde do trabalho, é possível identificar falhas e deficiências que estes locais por ventura apresentem.

Nesse contexto, este trabalho apresenta o desenvolvimento de uma metodologia participativa para análise das condições de segurança em um laboratório de ensaios de matérias e equipamentos utilizados em redes de distribuição de energia elétrica. Esta visa permitir a caracterização de possíveis ações de melhoria para eliminar os riscos, ou ao menos reduzi-los a índices mínimos e aceitáveis. A importância do caráter participativo foi também evidenciada por Salim e Carvalho (2002), segundo os autores os trabalhadores devem participar da identificação dos riscos, pelo fato de estarem envolvidos de forma direta no processo. Estes até podem não saber as causas dos problemas da falta de segurança, mas sofrem suas conseqüências.

\section{Metodologia}

A metodologia de trabalho baseou-se em uma abordagem ergonômica de situação do problema, composta de 4 etapas discriminadas essencialmente como: Análise da Demanda, Observação ou apreciação ergonômica, Construção de uma ferramenta de análise e Aplicação da ferramenta.

A Análise da Demanda foi realizada através de consultas às supervisões do laboratório, bem como os trabalhadores, que foram ouvidos para identificar as reais necessidades do setor. Também foram analisados os indicadores de segurança e saúde disponíveis no laboratório, para auxiliar na identificação dos postos mais problemáticos, ou as atividades mais penosas para os trabalhadores. Além disso, também foi observado o histórico do setor, identificando medidas já implantadas para melhorar as condições de segurança e saúde dos trabalhadores.

$\mathrm{Na}$ seqüência, foi realizada uma observação ergonômica analisando e confrontando as dimensões dos processos de ensaio do ponto de vista técnico, com a opinião dos laboratoristas a respeito das condições de trabalho existentes, através de entrevistas assistemáticas. Porém, antes de efetuá-las deve-se explicar aos trabalhadores o conteúdo e o motivo do trabalho realizado, evidenciando sua participação como fundamental para o sucesso da aplicação da ferramenta. Por este aspecto, deve-se também questionar o trabalhador, no próprio local de trabalho, sobre as condições de segurança e saúde existentes no seu trabalho. Esta fase de reconhecimento foi 
realizada procurando evidenciar todos perigos existentes no ambiente.

Com base nas observações realizadas e na literatura pertinente partiu-se para a montagem de uma ferramenta de verificação das condições de segurança do laboratório. Os itens considerados basearam-se nas Normas Regulamentadoras do Ministério do Trabalho e Emprego e nas Normas da ABNT - NBR 9077 e NBR 12633, para complementar os itens referentes à proteção contra incêndio, por exemplo. Inicialmente, a construção da ferramenta privilegiou a atividade de coleta de dados, criando uma padronização dos resultados, de tal forma que os dados possam ser comparados ao longo do tempo, indicando melhorias na segurança e saúde do trabalho do local analisado. Em seguida, sua elaboração contemplou as disposições necessárias à empresa para poder identificar possíveis falhas na segurança e saúde do local. Finalmente, a possibilidade de indicação de medidas de controle ou eliminação dos riscos. No que concerne aos procedimentos de aplicação, tomou-se o cuidado para que a ferramenta fosse o mais objetiva possível, caso contrário prejudicaria a agilidade na coleta de dados que deve ser peculiar deste tipo de avaliação.

Sendo assim, de posse das 32 Normas Regulamentadoras do Ministério do Trabalho, pôdese construir um inventário abordando as principais disposições legais e necessárias para manutenção da segurança e saúde dos trabalhadores. Para sua essência constitutiva, guardou-se uma atenção especial para não misturar tópicos e itens de normas. Estes necessitam seguir uma ordem lógica, partindo da situação macro (condições ambientais e das instalações) até chegar a uma análise micro, na qual são observados os indivíduos e as tarefas por eles executadas. Desta forma, a primeira coluna da ferramenta é destinada aos itens das normas, numerados de forma crescente para facilitar a procura por tópicos. Pode-se observar que os itens das normas foram descritos de forma resumida, proporcionando para o analista uma rápida leitura do item a observar. A segunda coluna é destinada à marcação da existência ou não do item indicado na primeira coluna. Caso se observe o atendimento à norma, marca-se um ' $\mathrm{X}$ ' na coluna SIM, caso contrário, marca-se um ' $\mathrm{X}$ ' na coluna NÃO.

Porém, podem existir situações em que a determinação da norma está atendida, mas não de forma completa. Por exemplo, verificar a existência do PPRA (Programa de Prevenção de Riscos Ambientais embora esteja disponível na Intranet, não está atualizado nem se encontra no local à disposição dos trabalhadores conforme determina a Norma Regulamentadora NR-9). Para estas situações, preenche-se a terceira coluna, onde é informada a adequação ou não deste item.

A quarta e última coluna da ferramenta de análise de condições de segurança e saúde é destinada aos comentários e/ou considerações sobre o item abordado, como por exemplo, a explicitação de uma não-conformidade, ou a indicação do local onde foi identificado o risco. Esta coluna deve ser preenchida sempre que o item correspondente não estiver de acordo com as condições de segurança e saúde preconizados pela NR, a fim de facilitar posteriormente a indicação 
da medida corretiva. A figura 1 apresenta um fragmento da estrutura do checklist com todos os itens utilizados para análise das condições ambientais de trabalho.

Figura 1: Estrutura da ferramenta

\begin{tabular}{|c|c|c|c|c|}
\hline \multirow{2}{*}{\multicolumn{2}{|c|}{ ITEM DE SEGURANÇA }} & \multirow{2}{*}{\begin{tabular}{|c|c|} 
EXISTE \\
SIM & NÃOO \\
\end{tabular}} & ADEQUADO & \multirow{2}{*}{ OBSERVAÇÕE } \\
\hline & & & \begin{tabular}{|l|l|} 
SIM & NÃO \\
\end{tabular} & \\
\hline 1. & Disposições Gerais - NR-1 & & & \\
\hline 1.1 & Ordens de Serviço sobre segurança e medicina do trabalho & & & \\
\hline 2. & Documentação básica para fiscalização & & & \\
\hline 2.1 & Mapa de riscos ambientais - CIPA & & & \\
\hline 2.2 & Pasta com o CA (Certificado de Aprovação) dos EPI utilizados na empresa & & & \\
\hline 2.3 & Prontuário de Instalações Elétricas, elaborado por profissional qualificado & & & \\
\hline 2.4 & $\begin{array}{l}\text { Prova de manter profissional qualificado e autorizado a trabalhar em } \\
\text { instalações elétricas }\end{array}$ & & & \\
\hline 2.5 & Livro de Registro de Seguranca do recipiente de gases e ar comprimido & & & \\
\hline 2.6 & Comprovante de treinamento da Brigada de Incêndio & & & \\
\hline 2.7 & Ficha de controle de inspeção dos extintores & & & \\
\hline 3. & Programa de Controle Médico de Saúde Ocupacional - NR-7 & & & \\
\hline 3.1 & $\begin{array}{l}\text { Programa de Controle Médico de Saúde Ocupacional - PCMSO, elaborado } \\
\text { por profissional legalmente habilitado }\end{array}$ & & & \\
\hline & $\ldots$ CONTINUA & & & \\
\hline
\end{tabular}

Após a construção da ferramenta, partiu-se para sua aplicação no local a ser analisado. Com a ferramenta em mãos, foram revistados todos os setores e postos de trabalho, observando todos os itens listados. Nesta fase, é importante o acompanhamento de supervisores dos setores em análise ou do pessoal do SESMT (Serviço Especializado em Segurança e Medicina do Trabalho), para facilitar os trabalhos de coleta de dados em termos de acesso aos locais e de dirimir dúvidas eventuais.

Ao aplicar a ferramenta, podem ser utilizadas fotos dos locais e das atividades observadas. Tal procedimento pode auxiliar na identificação do setor e das instalações existentes. Já as fotos concernentes às tarefas, podem auxiliar na descrição destas, permitindo ilustrar os processos de trabalho. Porém, a aplicação do registro fotográfico só deve ser realizada com o consentimento prévio da empresa e dos trabalhadores envolvidos.

A etapa final desta metodologia consiste na indicação das ações necessárias para implantar as melhorias nas condições de trabalho e segurança no laboratório. A fase seguinte da aplicação da ferramenta elaborada consiste em um Plano de Ação, ressaltando os itens indicados como não existentes ou não adequados na primeira parte do documento, por exemplo: Como será solucionada esta não-conformidade? Quem será responsável por esta ação? Por que é necessário realizar esta modificação? Quanto irá custar e quando será realizada? De forma ilustrativa, estas indagações no Plano de Ação estão intituladas por: ITEM DE SEGURANÇA; COMO?; QUEM?; POR QUÊ?; QUANTO?; QUANDO?, respectivamente. A figura 2 apresenta um fragmento da segunda etapa da ferramenta com todos os itens utilizados para a indicação do plano de ação das melhorias.

As informações referentes à área técnica, colunas intituladas ITEM DE SEGURANÇA, COMO? e POR QUÊ?, devem ser preenchidas pelo analista. De posse destes dados, o analista pode 
marcar uma reunião com os atores da empresa envolvidos diretamente na resolução dos problemas identificados, por exemplo: área gerencial dos setores analisados, representantes do SESMT (Serviço Especializado em Segurança e Medicina do Trabalho) e responsáveis pela área de meio ambiente da empresa. Nesta reunião, o analista apresenta os pontos identificados como nãoconformes, fazendo uma explanação detalhada de cada item para a total compreensão de todos os presentes, visto que, muitos integrantes desta reunião, são leigos na área. $\mathrm{O}$ analista deverá apresentar uma lista de priorização para resolução dos itens. Esta deve ter como base o conhecimento técnico do analista a cerca do problema, identificando possíveis falhas capazes de gerar maior risco à saúde e à segurança dos trabalhadores. Neste caso, pode ser utilizada uma escala de prioridade de execução: de 1 a 10, onde de 1 a 3 é baixa: de 4 a 6 é média e, por fim, de 7 a 10 é alta. O preenchimento das demais colunas, QUEM, QUANTO e QUANDO, ficam a cargo dos representantes da empresa. Esta última necessita de uma atenção especial da empresa, de acordo com a priorização do analista.

Figura 2 - Estrutura do Plano de Ação

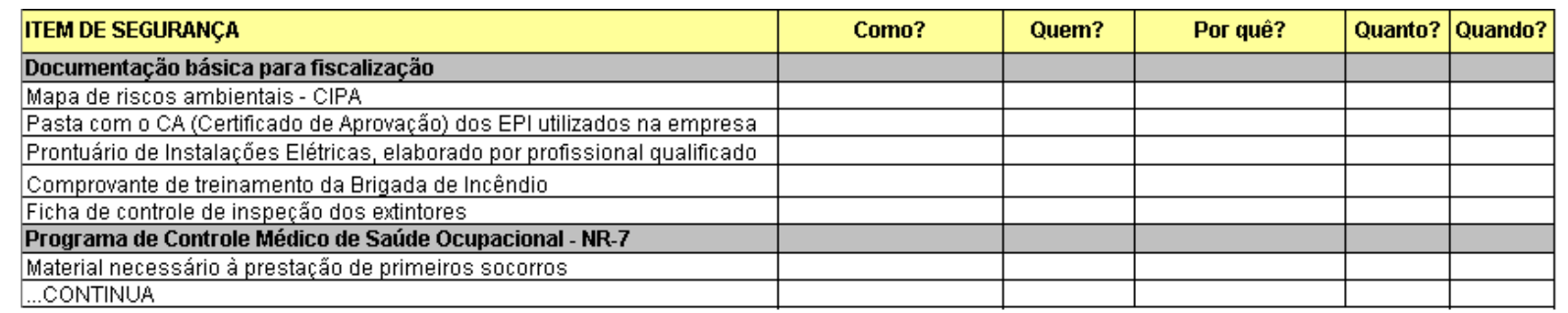

\section{Resultados}

A metodologia de análise foi aplicada no laboratório de ensaios de materiais e equipamentos utilizados para construção e manutenção de redes de energia elétrica em uma empresa de distribuição de energia elétrica. No laboratório são realizados ensaios mecânicos, químicos e elétricos para aferição da qualidade dos materiais e equipamentos utilizados nas redes de energia elétricos. O laboratório está localizado em uma unidade separada dos demais setores da empresa, dentro das instalações do seu operador logístico, com uma área de $134 \mathrm{~m}^{2}$ separados em três salas. O piso possui revestimento vinílico semiflexível e as paredes externas são de tijolo maciço aparente e as divisórias internas modulares.

$\mathrm{Na}$ Análise da Demanda foi realizado um levantamento das atuais condições de saúde e segurança das instalações do laboratório, tendo como base dados coletados das entrevistas com os supervisores e os operadores do laboratório. Não foram analisados dados referentes aos acidentes e afastamentos ocorridos ou as informações contidas no PPRA (Programa de Prevenção de Riscos Ambientais) e PCMSO (Programa de Controle Médico de Saúde Ocupacional) do laboratório, já 
que estes dados não estavam disponíveis de forma atualizada. As informações pesquisadas indicaram que o laboratório apresenta um nível satisfatório de segurança, porém aquém de suas necessidades. Neste setor são realizados ensaios onde são manipulados produtos e equipamentos capazes de gerar grande risco para a integridade física e fisiológica dos trabalhadores.

$\mathrm{Na}$ segunda etapa de análise, após a observação e apreciação da situação existente, no que concerne aos processos e às condições de trabalho, constatou-se uma mistura de ensaios de características diferentes serem realizados no mesmo ambiente, por exemplo, processos químicos e mecânicos. No que diz respeito aos processos elétricos, em razão de sua especificidade e periculosidade, estes eram mais isolados. Quanto aos laboratoristas, as entrevistas indicaram certo desconhecimento da dimensão de periculosidade dos processos por eles tratados, principalmente no que diz respeito aos aspectos: ergonômicos, de organização do trabalho, de utilização de EPI (Equipamentos de Proteção Individual) e EPC (Equipamentos de Proteção Coletiva), bem como fatores de risco de segurança em geral.

Após a construção da ferramenta esta foi aplicada em conjunto com os laboratoristas onde foi possível verificar o atendimento ou não das normas do Ministério do Trabalho. Com o documento em mãos, foram seguidos verificados e analisados todos os seus itens em todas as instalações do laboratório. Em alguns casos, em especial aqueles referentes à documentação, foi necessário também consultar o supervisor do laboratório para verificar o atendimento destes itens. Quanto aos operadores, estes participaram em situações mais específicas, para assegurar que determinado item estava realmente sendo atendido; por exemplo, quando foi necessário saber se eles dominavam as técnicas de extinção do fogo por meio de extintores.

De outra forma, observou-se que em alguns aspectos, principalmente aqueles relacionados à resposta imediata sobre perigos ou condições de conforto e higiene no local, há necessidade de melhorias imediatas. Neste sentido, os principais itens não-conformes identificados no laboratório analisado concernem aos treinamentos e capacitações dos trabalhadores do laboratório para a correta utilização dos sistemas de combate ao fogo e técnicas de primeiros socorros. Os funcionários nunca receberam um treinamento formal com o objetivo de instruí-los sobre as técnicas de manuseio de extintores, classes do fogo, tipos de cargas dos extintores e seu uso correto. Também não foram informados sobre os procedimentos que devem ser adotados em caso de um incêndio nas instalações da empresa de transportes, onde está localizado o laboratório e as rotas de fuga mais seguras. Com relação aos procedimentos de primeiros socorros, além de não terem um treinamento específico que os habilitem a atuar como socorristas, o laboratório não dispõe de um kit com itens básicos para atendimento de vítimas de acidentes.

Os sanitários existentes para o uso dos funcionários do laboratório, apesar de serem separados por sexo, terem o número de chuveiros e vasos sanitários suficientes para a população 
usuária, como determina a NR-24, estão distantes do local de trabalho (em torno de $150 \mathrm{~m}$ ). Estes também não apresentaram boas condições de higiene; constatação confirmada pelos funcionários, que também foram questionados sobre este tópico, respondendo ser comum encontrarem os banheiros em tal estado. Em termos de instalações do laboratório, estas atendem o disposto na NR25, onde são determinadas medidas para a correta destinação dos resíduos gerados, havendo a correta separação do lixo, sendo disponibilizadas lixeiras específicas e identificadas. De outra forma, observou-se a falta de espaço para acomodação de todos os equipamentos e máquinas utilizados e necessários para realização dos ensaios. Este fato ocasiona o não atendimento aos itens da NR-12, que determinam as distâncias mínimas de segurança entre máquinas e a delimitação de áreas exclusivas de circulação.

Os sistemas de proteção e combate contra incêndio do local apresentaram, em sua maioria, deficiências. Os extintores existentes no local não eram suficientes para atender toda a área do laboratório e também os menos indicados para a classe de fogo existente no local. Além disso, eles se encontravam obstruídos por caixas ou mesas, dificultando o acesso em caso de emergência e não estavam sinalizados por placas fixadas na parede, o que facilitaria sua visualização de qualquer ponto do laboratório. Contudo, as unidades extintoras existentes no local estavam dentro do prazo de validade, com pressão para o uso e selo do INMETRO.

Com relação aos Equipamentos de Proteção Individual (EPI), estes são fornecidos para os trabalhadores do laboratório, porém não foram treinados sobre o uso, higienização e guarda correta dos EPI. Também não foram identificados cartazes indicativos visíveis salientando o uso obrigatório destes equipamentos. Outro aspecto não atendido, está relacionado ao local para guarda dos EPI. Neste caso, a empresa deveria disponibilizar armário individual para cada funcionário, onde este possa guardar seus equipamentos de forma organizada.

Os aspectos relacionados ao conforto no ambiente de trabalho, determinados pela NR-17, são atendidos de forma parcial. Os funcionários não procedem de forma adequada as corretas técnicas de manuseio e transporte de cargas utilizadas em equipamentos e materiais de ensaio, observando que a capacitação não obteve efetividade. Também não são utilizados meios de transporte auxiliares para facilitar a movimentação de cargas. De outra forma, alguns postos de trabalho não apresentaram espaço suficiente para a movimentação adequada dos diversos segmentos corporais e as mesas dos postos para uso de computadores não são dotadas de regulagens.

A partir do reconhecimento das condições físico-ambientais do laboratório, utilizando-se para tal a ferramenta apresentada anteriormente, foi necessário então elaborar um plano de ação para resolver as deficiências e os problemas identificados relacionados à segurança e saúde no trabalho. Nesta etapa, coube ao analista discutir com os gerentes do setor analisado e os integrantes 
do SESMT (Serviço Especializado em Segurança e Medicina do Trabalho) quais itens deveriam ser priorizados, segundo um escala de risco estabelecido pelo próprio analista e a disponibilidade temporal e financeira de execução das medidas corretivas por parte da empresa.

No estudo realizado neste trabalho, uma das melhorias sugeridas, que ao ser executada, poderia solucionar vários problemas constatados no laboratório, seria a ampliação de suas instalações. Com esta medida, o laboratório teria mais espaço para a acomodação de todos os equipamentos e máquinas utilizados, respeitando o distanciamento mínimo requerido pelas normas para garantir a segurança dos operadores e dos demais usuários que transitam entre os setores. $\mathrm{O}$ aumento de área auxiliaria na distribuição dos extintores, que teriam um local fixo e devidamente sinalizado, evitando obstruções e mudança arbitrária destes sistemas de proteção para facilitar as demais atividades.

Com relação às instalações sanitárias existentes, a empresa deveria disponibilizar banheiros mais próximos ao local de trabalho. Porém, uma medida que necessita ser atendida prontamente é a limpeza eficaz dos banheiros existentes, uma vez que as condições de falta de higiene constatadas no local, podem gerar riscos diretos à saúde dos trabalhadores.

Quanto aos equipamentos de proteção, a empresa deverá treinar e capacitar todos os envolvidos no laboratório sobre o uso adequado, modos de higienização e guarda. Deverá ainda disponibilizar armários individuais para cada funcionário, de maneira que estes possam guardar seus EPI de forma organizada. Não há como cobrar boas práticas de uso de EPI se não forem fornecidas condições mínimas de higiene e organização para tal.

\section{Conclusão}

Este estudo permitiu, de forma participativa, identificar, reconhecer, avaliar e sugerir melhorias de segurança e saúde do trabalho em um laboratório de ensaios de equipamentos e materiais utilizados na construção e manutenção de redes de distribuição de energia elétrica, a partir da construção e posterior aplicação de uma metodologia abordando diversos aspectos relacionados às Normas Regulamentadoras do Ministério do Trabalho. Neste âmbito, a consulta e a participação dos supervisores e trabalhadores relacionados diretamente com as atividades fortaleceu ainda mais os resultados obtidos na aplicação da metodologia.

Com a construção da metodologia abordada neste trabalho, foi possível identificar, de forma rápida e precisa, condições inadequadas de segurança e saúde nos demais setores da empresa onde o estudo foi aplicado. Salvo algumas alterações ou complementações, esta metodologia pode ser utilizada em outros seguimentos industriais, auxiliando profissionais ligados às áreas de segurança e medicina do trabalho na identificação de não-conformidades às Normas Regulamentadoras do 
Ministério do Trabalho. Além disso, a metodologia empregada na fase de sugestões de melhorias, procurou reunir os responsáveis diretos e com poder de decisão para agilizar as modificações necessárias. Estes foram essencialmente os gerentes do setor analisado, os integrantes do SESMT (Serviço Especializado em Segurança e Medicina do Trabalho) e da área de Meio Ambiente da empresa. Salienta-se também que muitas das medidas apresentadas, não dependem de grandes investimentos financeiros, mas sim de uma mudança de postura e conscientização dos trabalhadores e de seus dirigentes sobre segurança e saúde no trabalho. Informações, palestras, campanhas e treinamentos muitas vezes são mais eficazes do que a mudança física das atuais instalações. Complementando, Iida (1990) indica que o conhecimento das situações perigosas e o desenvolvimento de um comportamento para evitá-las podem diminuir significativamente os acidentes, principalmente quando se trata de controles ambientais de riscos onerosos, que dificilmente serão instalados pela empresa.

De outra forma, constata-se que este estudo analisou somente tarefas (situações estáticas), apenas através da observância do atendimento de normas, não considerando os riscos gerados pelas atividades desenvolvidas dentro do laboratório. Para a evolução futura deste trabalho, pode-se vislumbrar então, como complemento essencial da metodologia, a inserção da análise das atividades, sugerindo a necessidade de novas pesquisas, que abordando a análise do trabalho real contemplará de forma mais completa a análise de riscos em laboratórios.

\section{Referências}

ASSOCIAÇÃO BRASILEIRA DE NORMAS TÉCNICAS. Requisitos gerais para a competência de laboratórios de ensaio e calibração - NBR ISO 17025. Rio de Janeiro: ABNT, 2005.

ASSOCIAÇÃO BRASILEIRA DE NORMAS TÉCNICAS. Saídas de emergência em edifícios - NBR 9077. Rio de Janeiro: ABNT, 1993.

ASSOCIAÇÃO BRASILEIRA DE NORMAS TÉCNICAS. Sistemas de proteção por extintores de incêndio - NBR 12633. Rio de Janeiro: ABNT, 1993.

BENOLIEL, M. J. Step-by-step implementation of a quality system in the laboratory. Tends in Analytical Chemistry, v.18, n.9, p. 632-638, 1999.

\section{cross ref}

BRASIL. Ministério do Trabalho e Emprego. Legislação: normas regulamentadoras. Disponível em: $<$ http://www.mte.gov.br/Empregador/SegSau/Legislacao/Normas>. Acesso em: 12 jan. 2006.

CALÇA, R. R. Avaliando o perigo para tornar trabalhos mais confiáveis deve-se recorrer à simulação e ensaios específicos. Revista Proteção, p. 44-45, 1999.

IIDA, I. Ergonomia: projeto e produção. São Paulo: Edgard Blücher, 2000.

INSTITUTO NACIONAL DE METROLOGIA. Critérios para o credenciamento de laboratórios de ensaios segundo os princípios das boas práticas de laboratório. Norma NIT - CICLA - 028. Brasília, 2003. 30p.

ISO/IEC - GUIDE 25. General requirements for competence of testing and calibration laboratories. 4 ed., 1997.

ORGANIZATION FOR ECONOMIC CO-OPERATION AND DEVELOPMENT. Series on principles of good laboratory practices and compliance monitoring. Paris, 1998. 44p.

SALIM, C. A.; CARVALHO, L. F. Saúde e segurança no trabalho: contextos e vertentes. Belo Horizonte: Fundacentro/Universidade Federal de São João Del Rei, 2002. 


\begin{abstract}
The work conditions about materials and equipments in test laboratories used on electricity distribution network exposed their operators to important risks concerning health and safety. This work aimed to elaborate a participatory tool enable to study these conditions, reducing accidents risks and occupational diseases in these laboratories. The methodology procedures were applied in four steps: demand analysis (consulting supervisors and workers with analysis of safety and health indicators); development of a specific analysis tool based on existent policies; its application in the workplace with an indication of improvements presented in the form of an action plan. As a result, the tool showed to be simple and efficient in characterizing harmful situations to health and physical integrity of the workers.
\end{abstract}

Key-words: Safety, Health, Risks, Laboratory, Electricity.

\title{
Dados dos autores:
}

Nome completo: Fernando Gonçalves Amaral

Filiação institucional: Universidade Federal do Rio Grande do Sul

Departamento: Engenharia de Produção e Transportes

Função ou cargo ocupado: Professor Adjunto

Endereço completo para correspondência: Av. Osvaldo Aranha, 99 - $5^{\circ}$ andar (Centro, Porto Alegre, RS, Brasil, CEP:90035-190):

Telefones para contato: $51-33084292 \quad 51-33083491$

e-mail:amaral@producao.ufrgs.br

*TODOS OS AUTORES PODEM SER ENCONTRADOS PELO ENDEREÇO DO AUTOR PRINCIPAL

Recebido para publicação em: 12/04/07

Aceito para publicação em: 04/06/07 